\title{
Análisis del impacto de la Actividad Solar y la variabilidad climática en la productividad apícola para el territorio cubano
}

\author{
Analysis of the impact of solar activity and climate variability in \\ productivity beekeeping Cuban territory
}

\author{
Sierra-Figueredo, Pablo; Pérez-Piñeiro, Adolfo; Durán-Zarabozo, \\ Odil; Marinero-Orates, Edgar Antonio; Zaldivar, Juan Manuel; Editor \\ Academico Prof. Dr. Carlos A. Zuniga-Gonzalez
}

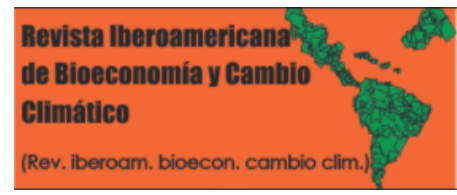

Pablo Sierra-Figueredo sierrafp@gmail.com

Instituto de Geofísica y Astronomía, AMA, CITMA Cuba, Cuba

(iD) Adolfo Pérez-Piñeiro

sierrafp@gmail.com

entro de Investigaciones Apícolas, Min. Agric. Cuba, Cuba

(D) Odil Durán-Zarabozo

sierrafp@gmail.com

Instituto de Geografía Tropical, AMA, CITMA, Cuba.

4.- Universidad de El Salvador., El Salvador

Edgar Antonio Marinero-Orates

sierrafp@gmail.com

Universidad de El Salvador., El Salvador

(D) Juan Manuel Zaldivar

sierrafp@gmail.com

Colegio de Post-Graduados en Tabasco, México.,

Mexico

Editor Academico Prof. Dr. Carlos A. Zuniga-

Gonzalez

Universidad Nacional Autnóma de Nicaragua, León.

Nicaragua, Nicaragua

Revista Iberoamericana de Bioeconomía y Cambio Climático

Universidad Nacional Autónoma de Nicaragua, León, Nicaragua ISSN-e: 2410-7980

Periodicidad: Semestral

vol. 1, núm. 2, 2015

czuniga@ct.unanleon.edu.ni

Recepción: 13 Julio 2015

Aprobación: 23 Diciembre 2015

URL: http://portal.amelica.org/ameli/journal/394/3941749003/

DOI: https://doi.org/10.5377/ribcc.v1i2.2481

Autor de correspondencia: sierrafp@gmail.com
Resumen: Los resultados que se muestran en el presente trabajo tratan de responder a algunas interrogantes surgidas en trabajos anteriores realizados por los mismos autores en cuanto a la incidencia de la actividad solar y geomagnética sobre la productividad apícola no sólo teniendo en cuenta la variabilidad multianual de las mismas, sino también la estacionalidad que de forma muy fuerte se observa en dicho renglón productivo y la intermodulación entre ésta y la variabilidad climática presente, también influida por la Actividad Solar. Para ello se llevó a cabo el procesamiento de la data melífera comprendida entre el año 1979 y el 2013 con resolución mensual, teniendo en cuenta la producción total de miel, la de cera y el rendimiento por colmena para todo el país. Como índice representativo del Clima Espacial se utilizó el índice geomagnético planetario Ap. Las series de tiempo generadas y analizadas, así como el resultado estadístico obtenido muestran nexos causales entre Ap y las variables apícolas dignos de tener en cuenta para una mejor planificación de la labor apícola con fines de mejorar la productividad..

Palabras clave: Clima Espacial, Actividad Solar, Productividad Apícola, Ap.

Abstract: The results shown in this paper try to answer some questions raised in previous work by the same authors in the incidence of solar and geomagnetic activity on productivity beekeeping not only taking into account the multi-year variability of the same but also the seasonality very strongly seen in this production line and intermodulation between it and present climate variability, also influenced by solar activity. It was carried out the honey processing data between 1979 and 2013 with monthly resolution, taking into account the total production of honey, wax and yield per hive for the whole country. As representative index Space Weather planetary geomagnetic index Ap used. The series generated and analyzed time as well as the statistical results obtained show causal links between Ap and worthy bee variables to consider for better planning of beekeeping work for the purpose of improving productivity.

Keywords: Space Weather, Solar activity, Beekeeping productivity, Ap. 


\section{INTRODUCCIÓN}

Las complejas relaciones existentes entre el Medio Ambiente y los procesos biológicos y otros que en el mismo se desarrollan constantemente, son temas que preocupan cada vez más debido al impacto que ello representa para la supervivencia de la vida en el planeta. Conociendo mejor dichas relaciones seremos capaces de optimizar la explotación de los recursos con el menor daño posible al medio ambiente que nos sustenta. (Babayev E.S. Crosby N.B., Obridko V.N. \& Rycroft M.J. 2008)

En este campo de acción se enmarca el trabajo que venimos realizando desde hace ya varias décadas $(1,2)$ y que esperamos que de alguna manera sea posible llevar a la práctica con una óptica regional que permita una mejor explotación no sólo de los recursos melíferos para la producción sino también para la mejor preservación de la población apícola, responsable en gran medida de la tan necesaria polinización que ellas realizan garantizando mayores producciones en nuestros campos. Todo ello, en modesta medida puede también contribuir a contrarrestar algunos de los dañinos efectos del cambio climático a que estamos, sin dudas sometidos. (E.W. Cliver, Y. Kamide, A.G, 2000)

Anteriormente Pérez A. y Sierra P, 2015.(3) mostraron la sensible relación entre el geomagnetismo terrestre y la productividad apícola para un largo período en Cuba. En dichos resultados se evidencia el sincronismo multianual entre dichas variables. Para esos resultados se utilizaron datos anuales solamente, de manera que la marcada estacionalidad de la producción apícola y la de 1 índice geomagnético no se evidenciaban, lo cual era necesario completar para una mejor comprensión del fenómeno, lo que lo hace más complejo debido a la intermodulación que se produce con la variabilidad climática cíclica.

Es importante también tener presente el comportamiento de la Iradiancia Solar (TSI) que se registra en la superficie del planeta, la que tiene un marcado comportamiento multianual regido por la Actividad Solar en sus ciclos undecenales (Fig. 1), lo que se debe ver reflejado en las variables climáticas y los procesos biológicos de la flora, último eslabón de la cadena nutricional de la población apícola.

Otros autores $(4,5)$ señalan en sus resultados la relación existente entre la Actividad Solar y diversos fenómenos del Clima y la Biosfera en general, haciéndose cada vez más firme la hipótesis de relación causal entre tan importantes fenómenos. (Pérez A., Sierra P., (1993)

\section{Materiales y Métodos.}

Para el desarrollo del presente trabajo utilizamos los datos de producción melífera mensual de toda Cuba (1979 - 2013), desglosados en provincias, incluyendo el rendimiento por colmena calculado, proporcionados por el Instituto de Investigaciones Apícolas del Ministerio de Agricultura. Los datos fueron tabulados adecuadamente en un libro EXCEL, donde fue incluida la serie temporal del índice geomagnético Ap promedio mensual obtenido de la base de datos geomagnéticos localizada enhttp://www.geomag.bgs.ac.uk/ cgi-bin/apindex, así como una columna con los valores máximos reportados de éste índice, el cual es representativo del estado de actividad Solar y su impacto en el planeta (clima espacial), siendo el mismo utilizado abundantemente para trabajos de este tipo. En todos los casos la producción de miel viene dada en $\mathrm{Tm}$, la de cera en $\mathrm{Kg}$ y el rendimiento en $\mathrm{Kg}$ /colmena. (Sierra F.P., Sierra F.S., Rodríguez T. R., Pérez P. A. 1999)

\section{NotAS DE AUTOR}


Se obtuvo las series de tiempo comparativas de todas las variables, lo que permite distinguir las posibles relaciones temporales entre ellas, realizando posteriormente otros análisis estadísticos que cuantifiquen las mismas. Utilizando las herramientas de cálculo del propio EXCEL y del MicrocalOrigin, obtuvimos otros resultados que se muestran más adelante. (Sierra P., Baca M. 2013)

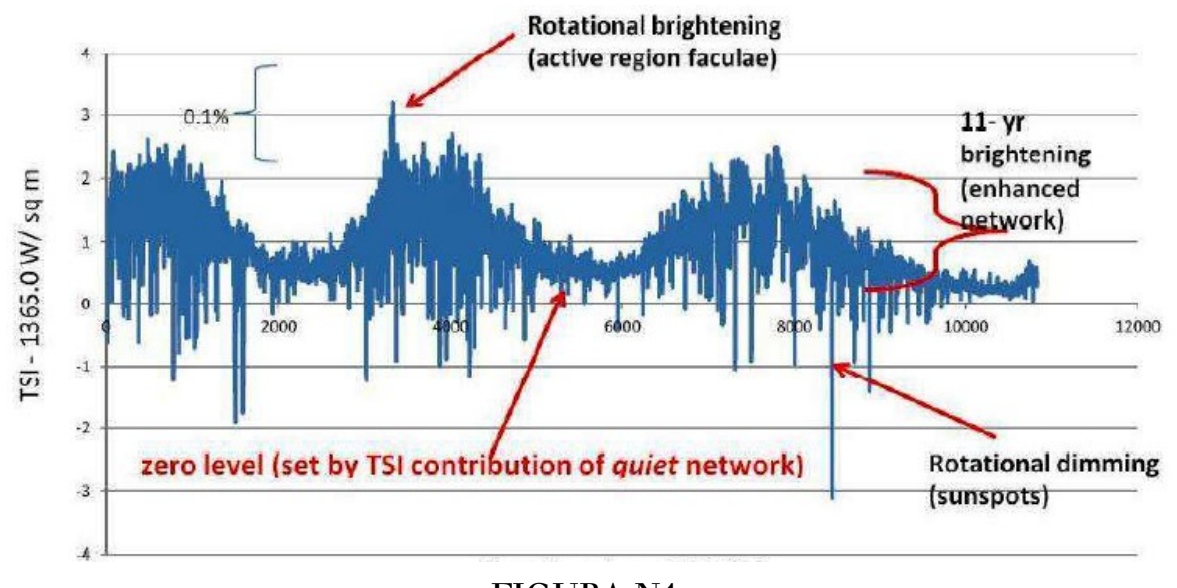

FIGURA N1

Comportamiento de la Irradiancia Solar desde 1978 y hasta el 2008, registrada por métodos radiométricos en el diapasón de 130 a $240 \mathrm{~nm}$ del espectro ultravioleta

Time (days since $11 / 17 / 78$ )

\section{Resultados}

En la Fig. 2 a, b, c,d se muestra las series de tiempo, con resolución mensual, del promedio del índice geomagnético Ap y de las variables apícolas.

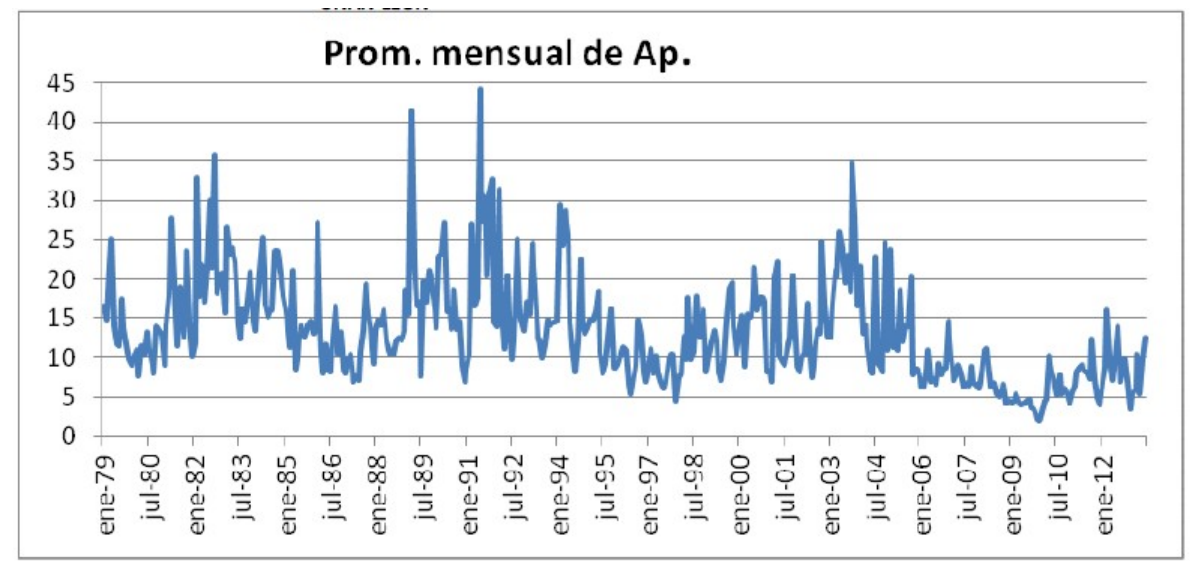

FIGURA 2A

Series de tiempo índice geomagnético Ap (a), de la producción de miel (Tm) (b),cera $(\mathrm{Kg})(\mathrm{c})$, rendimiento por colmena $(\mathrm{Kg} /$ colmena $)(\mathrm{d})$. En todos los casos son promedios mensuales. La serie comienza en enero de 1979 y concluye en marzo del2013.

(a) 


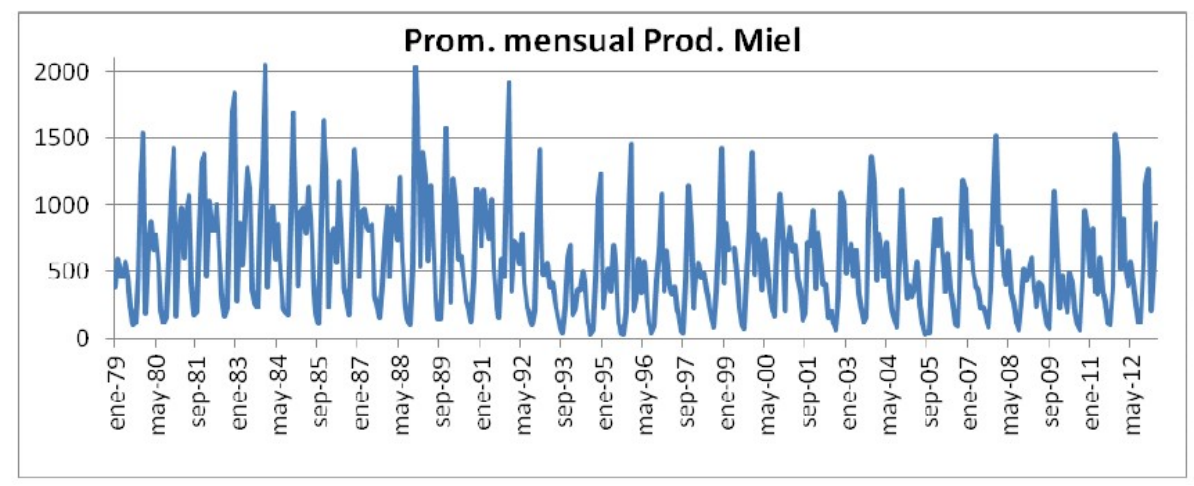

FIGURA 2B

Series de tiempo índice geomagnético Ap (a), de la producción de miel (Tm) (b),cera $(\mathrm{Kg})(\mathrm{c})$, rendimiento por colmena $(\mathrm{Kg} /$ colmena $)(\mathrm{d})$. En todos los casos son promedios mensuales. La serie comienza en enero de 1979 y concluye en marzo del2013. Promedio mensual de producción de miel

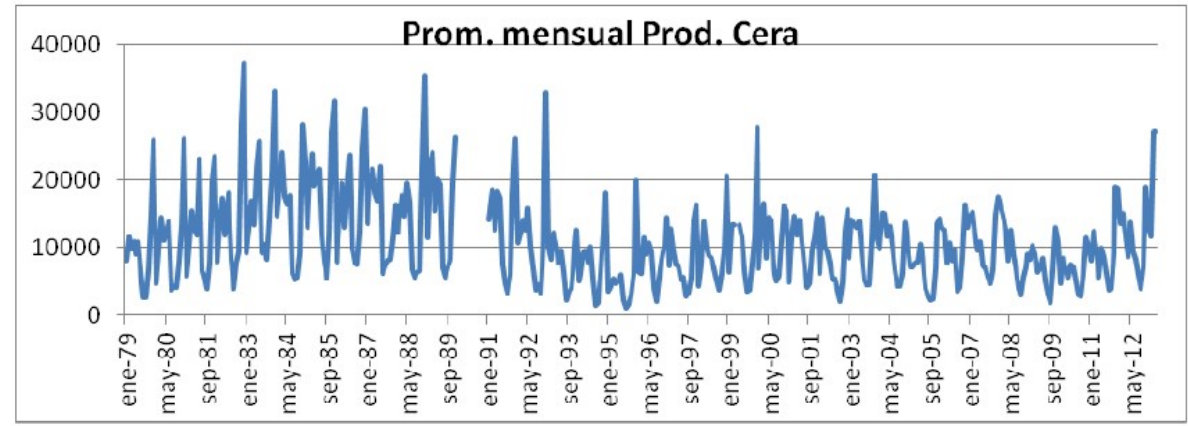

FIGURA 2C

Series de tiempo índice geomagnético Ap (a), de la producción de miel (Tm) (b),cera $(\mathrm{Kg})(\mathrm{c})$, rendimiento por colmena $(\mathrm{Kg} /$ colmena $)(\mathrm{d})$. En todos los casos son promedios mensuales. La serie comienza en enero de 1979 y concluye en marzo del2013. Promedio mensual de producción de cera

\section{FIGURA 2D}

Series de tiempo índice geomagnético Ap (a), de la producción de miel (Tm) (b),cera $(\mathrm{Kg})(\mathrm{c})$, rendimiento por colmena $(\mathrm{Kg} /$ colmena $)(\mathrm{d})$. En todos los casos son promedios mensuales. La serie comienza en enero de 1979 y concluye en marzo del2013.

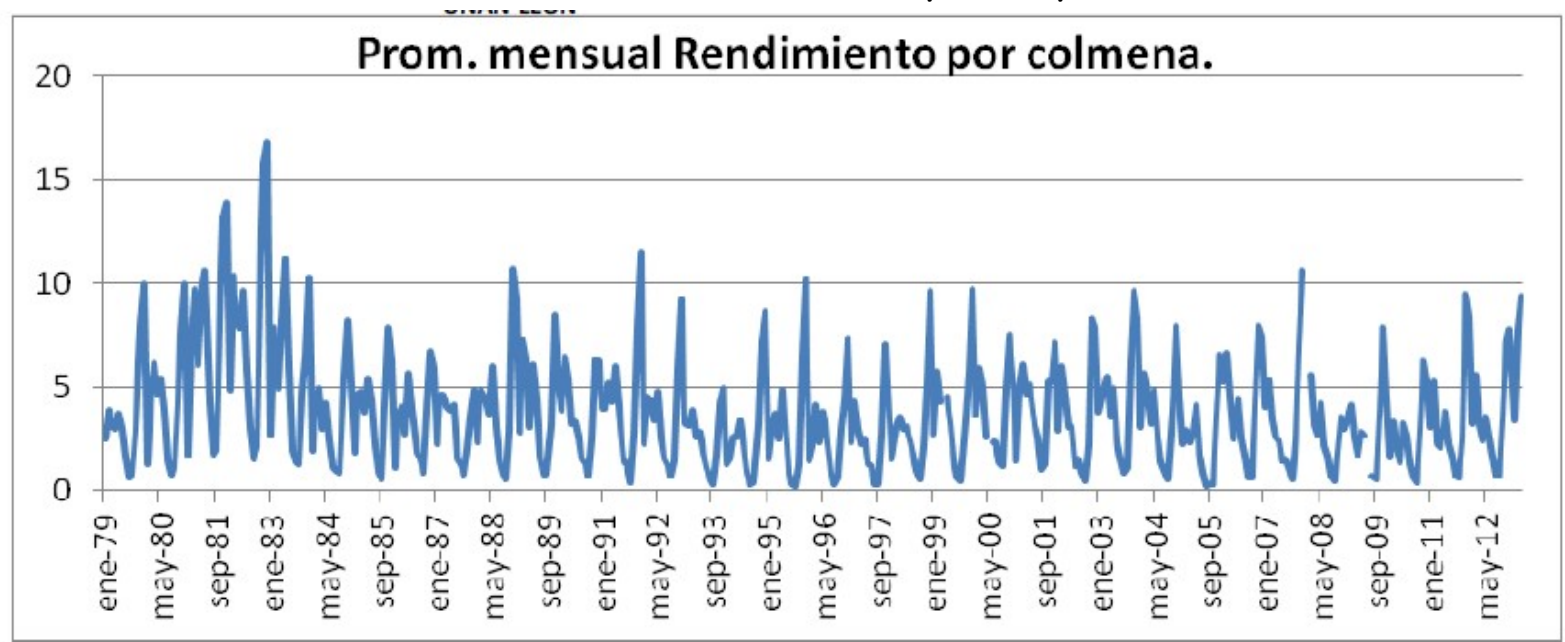


Fig. 2 Series de tiempo índice geomagnético Ap (a), de la producción de miel $(\mathrm{Tm})(\mathrm{b})$, cera $(\mathrm{Kg})(\mathrm{c})$, rendimiento por colmena $(\mathrm{Kg} /$ colmena) (d) . En todos los casos son promedios mensuales. La serie comienza en enero de 1979 y concluye en marzo del 2013.

Lo primero que podemos observar es la ciclicidad cuasi-undecenal ( 10 a 12 años) del índice Ap, lo cual es bien conocido en la literatura y por otra parte la marcada estacionalidad en las variables apícolas mostradas, lo cual es también conocido en trabajos precedentes citados.

Para poner de manifiesto esta última característica y corroborar su presencia también en el índice Ap, se hizo un análisis estacional para todas las variables durante los 34 años contemplados, encontrando el resultado que mostramos en la Fig. 3 a, b. el que se corresponden con lo reportado por Cliver et al. (2002) $(6,7)$ y se atribuye al balanceo del eje de rotación del planeta durante el año al realizar su viaje al rededor del Sol.

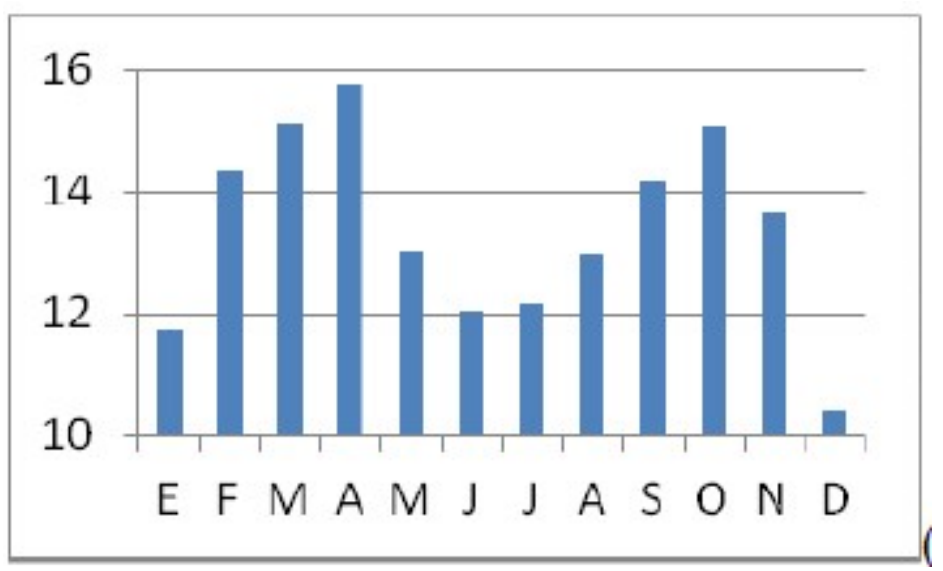

FIGURA 3A

Estacionalidad de Ap

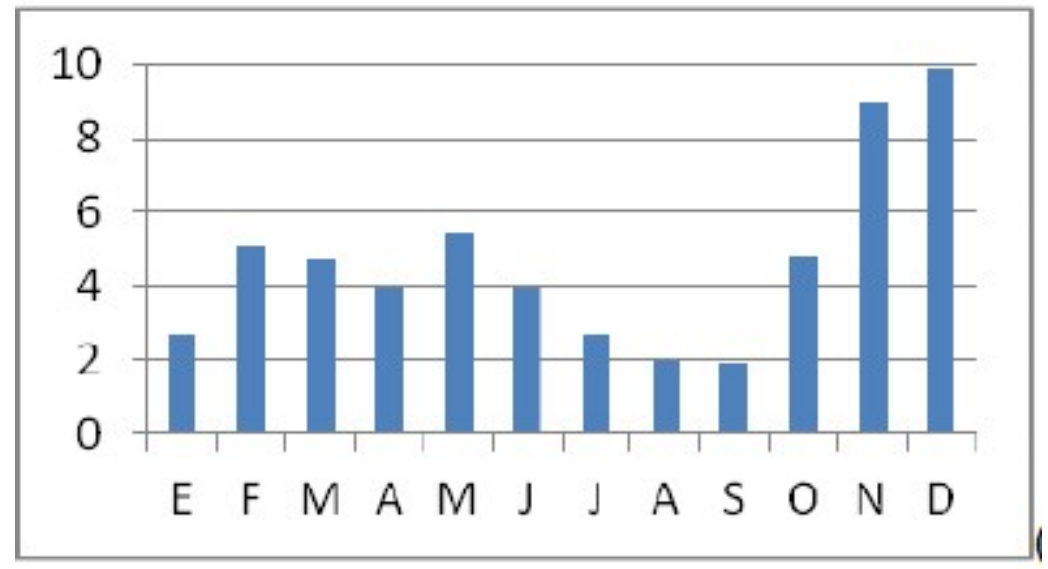

FIGURA 3B

Estacionalidad de Rendimiento para el período analizado b

Fig. 4 a, b, c, d, e, f, g. Comportamiento del Rendimiento mensual promedio para todo el período investigado. Se han superpuesto los meses para todos los años, obteniendo como resultado la tendencia predominante para el período para cada mes. La línea punteada roja representa el promedio para cada mes durante todo el período analizado. 


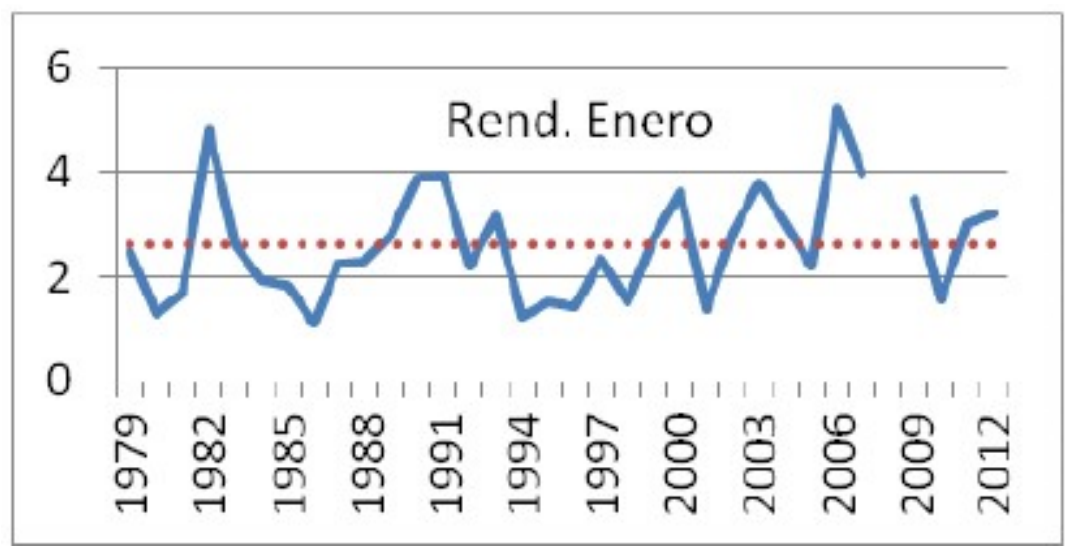

FIGURA 4A

Rendimiento según el mes del año

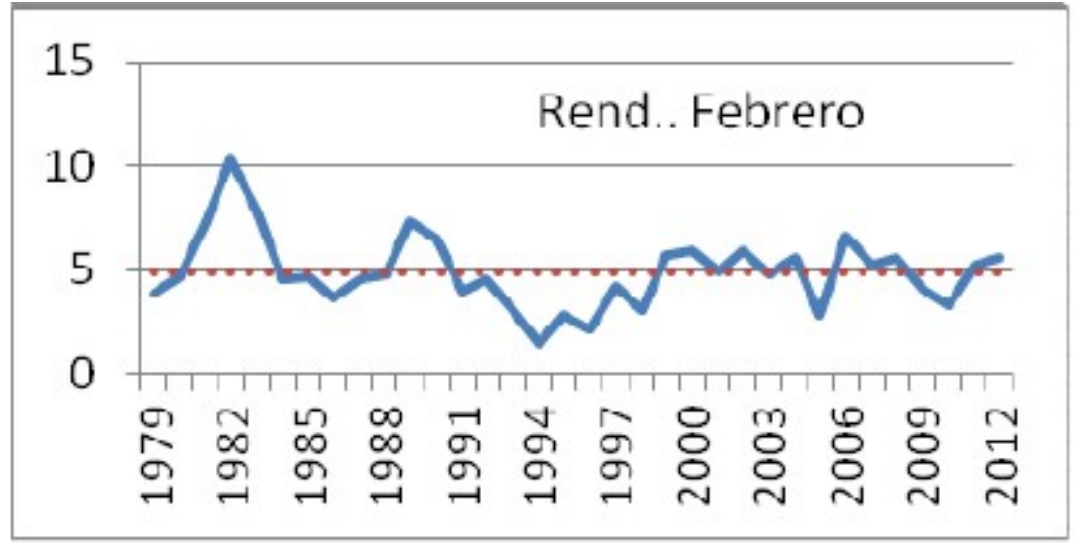

FIGURA 4B

Rendimiento según el mes del año

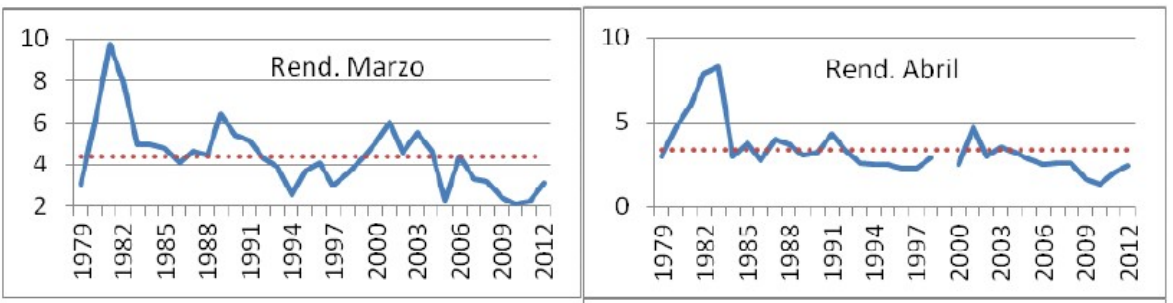

FIGURA 4C

Rendimiento según el mes del año,

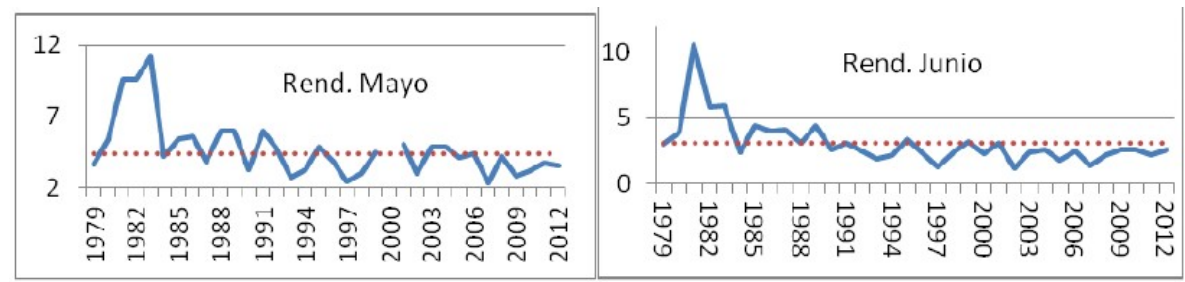

FIGURA 4D

Rendimiento según el mes del año, 


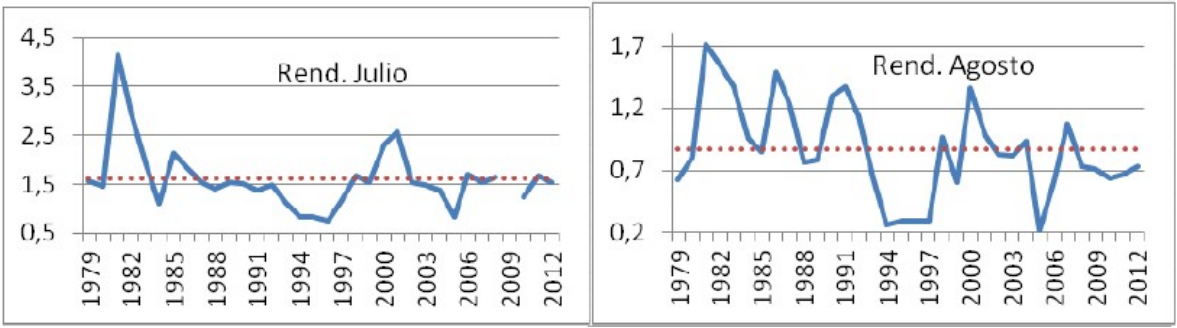

FIGURA 4E

Rendimiento según el mes del año,
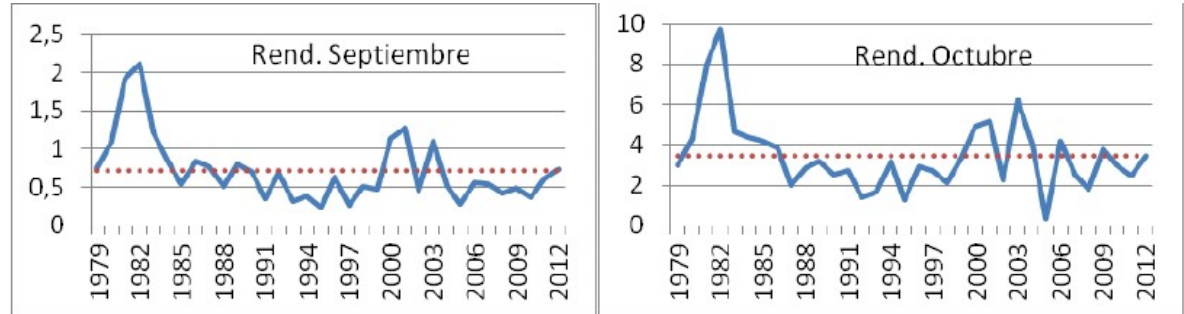

FIGURA 4F

Rendimiento según el mes del año,

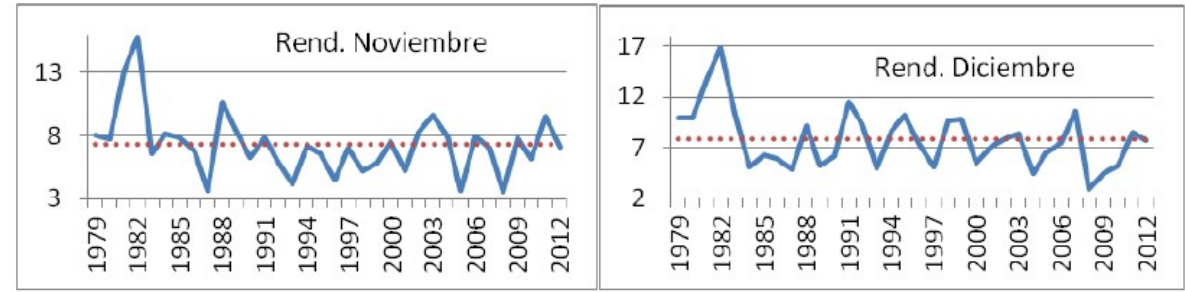

FIGURA 4G

Rendimiento según el mes del año

Fig. 4 Comportamiento del Rendimiento mensual promedio para todo el período investigado. Se han superpuesto los meses para todos los años, obteniendo como resultado la tendencia predominante para el período para cada mes. La línea punteada roja representa el promedio para cada mes durante todo el período analizado.

En la Fig. 5 se muestra la Estacionalidad de la Producción de Miel y de Cera para todo el período mediante superposición de los meses.

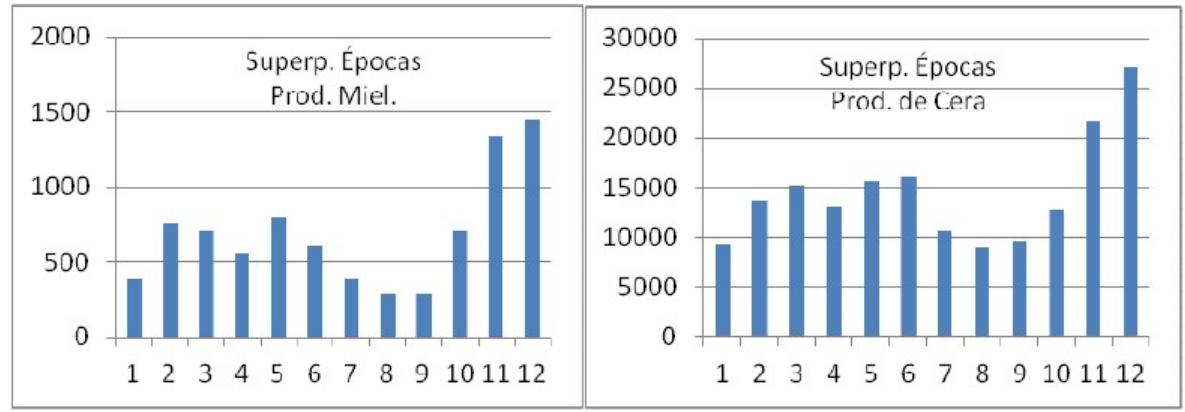

(a)

(b)

FIGURA 5

Estacionalidad de Producción de Miel (a) y Cera (b) para todo el período analizado. 
Fig. 5 Estacionalidad de Producción de Miel (a) y Cera (b) para todo el período analizado.

Y en la Fig. 6 se muestra los acumulados anuales (promedios) para Rendimiento (a) y para Ap (b), donde se evidencia el ciclo cuasi-undecenal en ambos gráficos.

Fig. 6 Comportamiento anual del índice Ap (b) y del Rendimiento (a) para todo el período analizado.

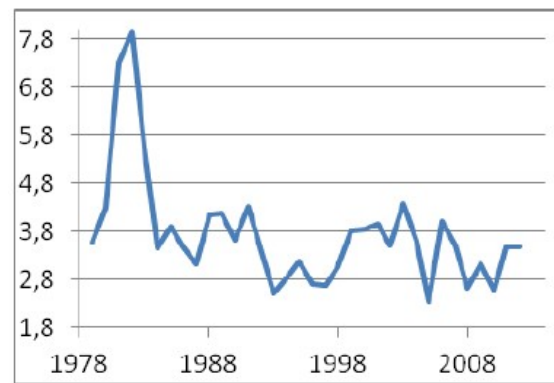

(a)

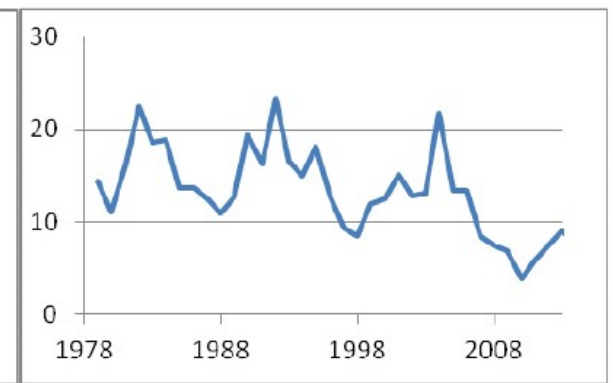

(b)

FIGURA 6

Comportamiento anual del índice Ap (b) y del Rendimiento (a) para todo el período analizado

Fig. 7 Se muestra un corte por trimestre, también utilizando una superposición de épocas para el Rendimiento por colmena.

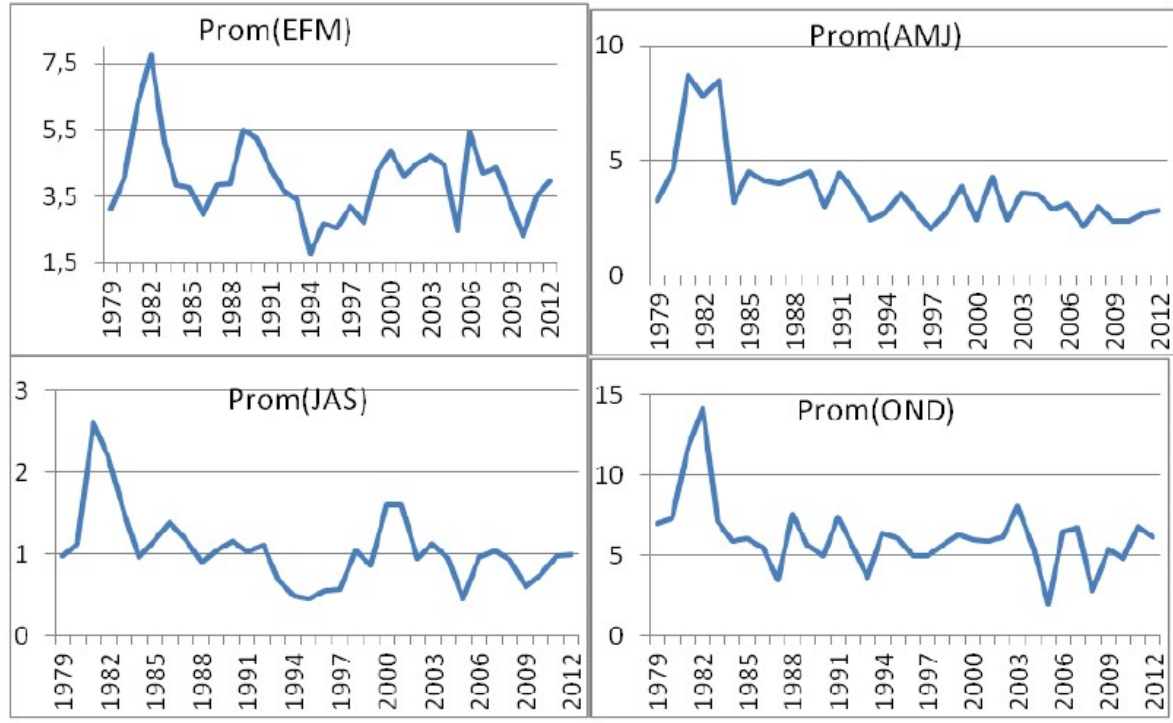

FIGURA 7

Se muestra un corte por trimestre, también utilizando una superposición de épocas para el Rendimiento por colmena.

Se obtuvo, mediante análisis de Regresión Lineal, la correlación entre el comportamiento anual de Ap y el Rendimiento apícola, con lo cual se encontró el resultado mostrado en la Figura 8. Para la producción de miel y cera se encontró resultados semejantes aunque con un coeficiente de correlación más bajo. 


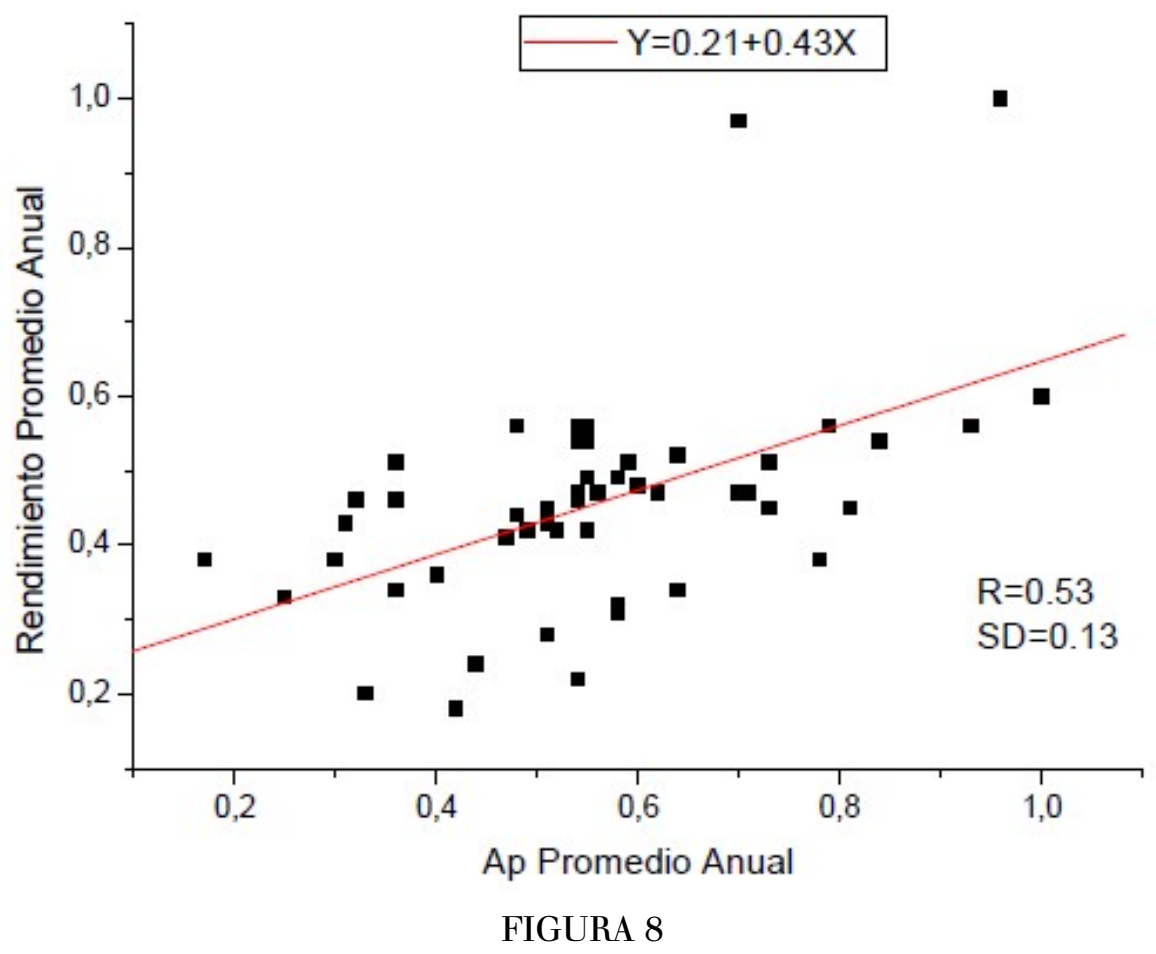

Regresión lineal entre Rendimiento promedio anual por colmena y valores promedios anuales de Ap para todo el período (1979-2013).

Un resumen cuantitativo de la asociación existente entre Ap y la productividad apícola viene mostrado en la Tabla $1 \mathrm{a}, \mathrm{b}$, donde se tabuló los períodos encontrados, condensado en la superposición de cada mes del año, lo cual también permite apreciar la diferencia existente entre diferentes épocas del año, tanto en el comportamiento de Ap como de la productividad, en cuanto a la compleja ciclicidad presente.

TABLA $1 \mathrm{~A}$

Períodos (en años y fracciones) espectrales presentes en la serie de tiempo del índice Ap promedio para cada mes del año después de realizar una superposición de épocas para todo el período estudiado

\begin{tabular}{cccccccc}
\hline ApProm.Anual & $\begin{array}{c}\mathbf{2 - 3} \\
\text { Años }\end{array}$ & $\begin{array}{r}\mathbf{3 - 4} \\
\mathbf{A n ̃ o s}\end{array}$ & $\begin{array}{c}\mathbf{4 - 5} \\
\text { Años }\end{array}$ & $\begin{array}{c}\mathbf{5 - 6} \\
\text { Años }\end{array}$ & $\begin{array}{c}\mathbf{6 - 7} \\
\text { Años }\end{array}$ & $\begin{array}{c}\mathbf{8 - 1 0} \\
\text { Años }\end{array}$ & $\begin{array}{c}\mathbf{1 0 - 1 6} \\
\text { Años }\end{array}$ \\
\hline $\mathbf{E}$ & & 3,$05 ; 3,76$ & & 5,33 & & & 16 \\
$\mathbf{F}$ & 2,7 & & & & & 8 & 16 \\
$\mathbf{M}$ & & & 4,6 & & & & 16 \\
$\mathbf{A}$ & & 3,37 & 4,27 & 5,3 & & 8 & \\
$\mathbf{M}$ & 2,7 & & 4 & 5,81 & & & \\
$\mathbf{J}$ & 2,$46 ; 2,9$ & & 4 & & 6,4 & & 10,$7 ; 16$ \\
$\mathbf{J}$ & 2,2 & 3,37 & & & 6,4 & & 10,$7 ; 16$ \\
$\mathbf{A}$ & 2,46 & & 4 & & 6,4 & & 10,7 \\
$\mathbf{S}$ & & 3,$04 ; 3,77$ & & 5,8 & & & 10,7 \\
$\mathbf{0}$ & 2,$4 ; 2,9$ & & 4 & & & & 10,$7 ; 16$ \\
$\mathbf{N}$ & & & & 6 & & & \\
$\mathbf{D}$ & 2,46 & 3,77 & & 5,8 & &
\end{tabular}

(a) 
TABLA 1 B

Rendimiento por colmena

\begin{tabular}{|c|c|c|c|c|c|c|c|}
\hline $\begin{array}{l}\text { Rendimiento } \\
\text { Prom.Anual }\end{array}$ & $\begin{array}{c}2-3 \\
\text { Años }\end{array}$ & $\begin{array}{c}\text { 3-4 } \\
\text { Años }\end{array}$ & $\begin{array}{c}4-5 \\
\text { Años }\end{array}$ & $\begin{array}{c}5-6 \\
\text { Años }\end{array}$ & $\begin{array}{c}\text { 6-7 } \\
\text { Años }\end{array}$ & $\begin{array}{l}8-10 \\
\text { Años }\end{array}$ & $\begin{array}{l}\text { 10-16 } \\
\text { Años }\end{array}$ \\
\hline $\mathbf{E}$ & & 3 & 4,3 & & 7,1 & 9,2 & \\
\hline F & & & 4,6 & 5,8 & & & 10,7 \\
\hline M & 2,7 & & & 5,3 & & & 10,$7 ; 16$ \\
\hline A & 2,7 & 3,6 & & & & 8 & 10,$7 ; 16$ \\
\hline $\mathbf{M}$ & 2,8 & & 4,0 & 5,8 & & 8 & \\
\hline $\mathbf{J}$ & 2,7 & & & 5,8 & & 8 & 12,8 \\
\hline $\mathbf{J}$ & 2,7 & 3,6 & & 5,8 & & & 12,8 \\
\hline A & 2,9 & & 4,3 & 5,3 & & & 16 \\
\hline $\mathrm{S}$ & 2,7 & & & 5,8 & & 8 & 10,$7 ; 16$ \\
\hline 0 & & 3,0 & & & & 8 & \\
\hline $\mathbf{N}$ & 2,9 & & 4,3 & & & 8 & \\
\hline D & & & 4,2 & & & & 12,8 \\
\hline
\end{tabular}

(b)

Tabla $1 \mathrm{La}$ tabla superior (a) resume los períodos (en años y fracciones) espectrales presentes en la serie de tiempo del índice Ap promedio para cada mes del año después de realizar una superposición de épocas para todo el período estudiado. (b) muestra el mismo resumen para los valores de Rendimiento por colmena. En ambos casos se separó por columnas los períodos cercanos agrupados de la forma mostrada en los encabezamientos de las tablas. El cálculo de los períodos se realizó mediante FFT utilizando el Microcal Origin.

Por cuanto la Actividad Solar y consecuentemente la Geomagnética no sólo hay que analizarlas en su carácter cíclico, sino también su comportamiento esporádico, pudiéndose presentar "eventos" tanto en la fase de alta actividad como en la de baja y en las transitorias, se hace necesario hacer una búsqueda del comportamiento, en este caso de la productividad apícola, alrededor de los cortos períodos de alta actividad para tener idea del verdadero impacto de un fenómeno en el otro. En nuestro caso la menor resolución temporal con que contamos es la mensual, por ello, tomamos, para los 34 años analizados, los 24 eventos geomagnéticos de mayor significación, representados por aquellos meses con valores máximos de "Ap Promedio" y de "Ap Máxima”. Se tabuló los valores mensuales correspondientes del mes de máximo valor, tres meses antes y seis meses posteriores, superponiendo los 24 "eventos", promediando y obteniendo el comportamiento estadístico característico, de lo cual se obtuvo como resultado el gráfico de la Fig. 9. De igual manera se tabuló, para los mismos períodos, las tres variables apícolas (Producción de Miel, de Cera y el Rendimiento), obteniendo como resultado el gráfico mostrado en la Fig. 10. En todos los casos los valores de las ordenadas son resultado de la normalización con el fin de estandarizar los gráficos. 


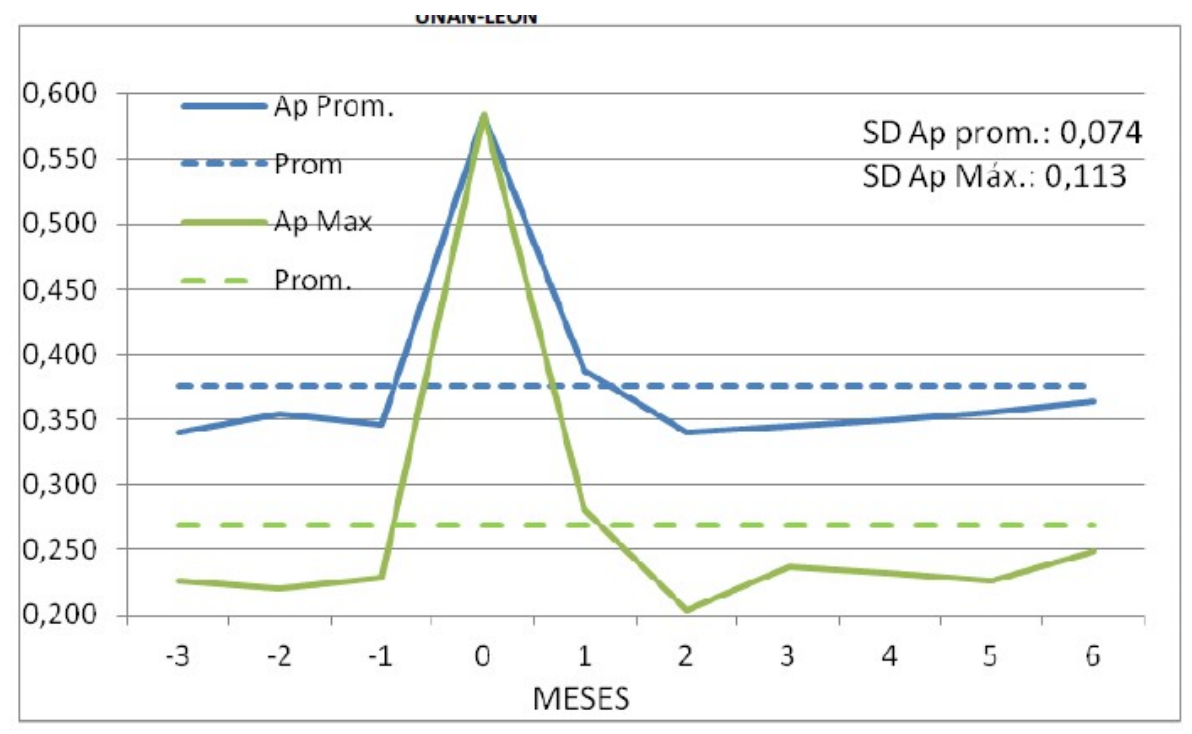

FIGURA 9

Resultado de la superposición de épocas para 24 "eventos” máximos de Actividad Geomagnética. El mes "0" es el de máximos valores seleccionados. Se muestran los valores de la Desviación Standard para ambas variables.

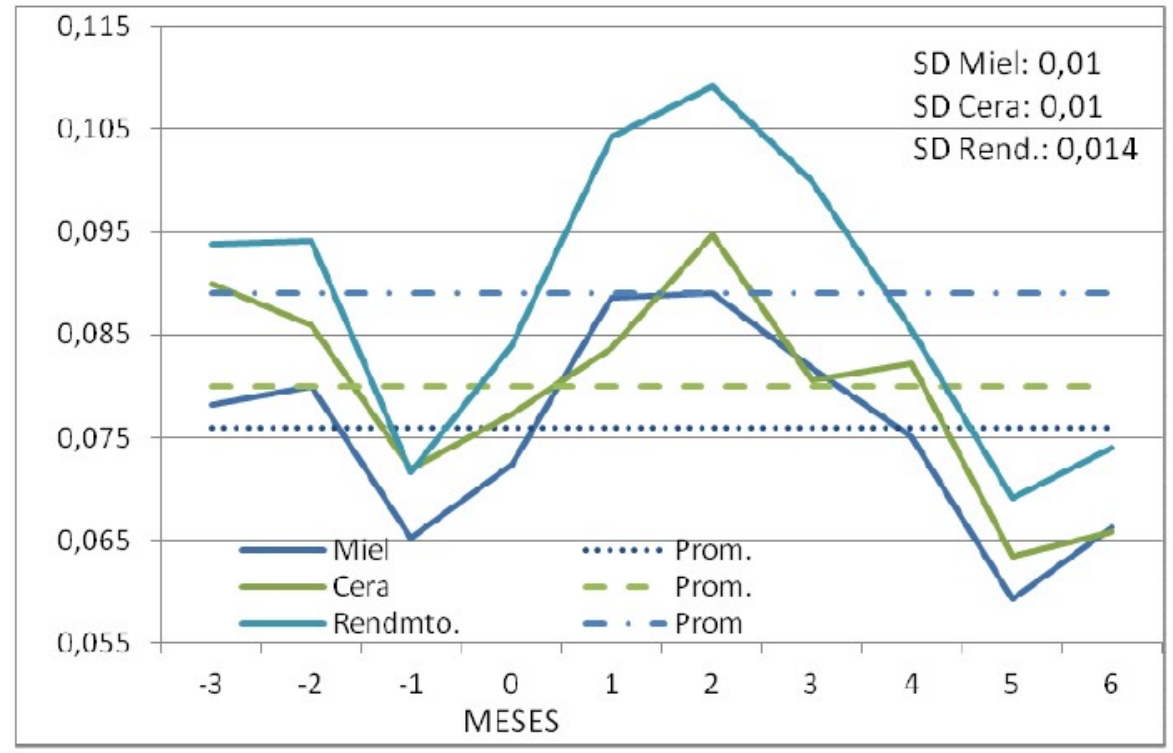

FIGURA 10

Resultado de la superposición de épocas para 24 "eventos” máximos de Actividad Geomagnética. El gráfico muestra el comportamiento estadístico de las variables apícolas alrededor de los meses con valores altos de Ap. El mes "0" es el de máximos valores de Ap seleccionados. Se muestran los valores de la Desviación Standard para las tres variables.

\section{Conclusiones y RECOMENDACIONES.}

De los resultados mostrados podemos llegar a algunas conclusiones, que si bien deben ser confirmadas para otros territorios, nos permiten corroborar resultados anteriores y afirmar que en las variables apícolas analizadas está presente la ciclisidad undecenal de la Actividad Solar y geomagnética, lo cual se evidencia en las Fig.1, 2, las que representan las series de tiempo con resolución mensual para Irradiancia Solar, Ap y 
las variables apícolas. Sin embargo, dicha ciclisidad se hace más evidente cuando observamos los gráficos de promedios anuales mostrados en la Fig. 6 y también es interesante observar cómo para diferentes meses del año dicha correlación se diferencia sustancialmente; por ejemplo, en la Fig. 4 se observa como en los meses de enero, febrero y marzo hay una mejor correlación multianual con el índice Ap, lo cual se muestra con mayor claridad cuando en la Fig. 7 se agrupan los datos por trimestres, siendo el primer trimestre el que mejor se ajusta a esta relación. En la Tabla 1 también encontramos la coincidencia de períodos iguales en varios de los meses.

Un aspecto interesante se refiere a la estacionalidad, tanto de Ap como de las variables apícolas. Las Fig. 3 y 5 muestran claramente dicha estacionalidad, muy marcada en todos los casos. En Ap, esta estacionalidad viene dada por el movimiento cíclico en la inclinación del eje terrestre durante cada año, lo que provoca que el viento solar, que permanentemente impacta al planeta, varíe con ese ritmo, lo cual el geomagnetismo terrestre lo manifiesta con la variabilidad observada, independientemente del grado de actividad solar cada año. Se puede observar en la Fig. 3 (a) cómo los valores máximos de Ap promedio anual se corresponden con los meses de primavera y otoño, esto es, en los equinoxios y los mínimos coinciden con los meses de verano e invierno (solsticios).

Observando la Fig. 3 (b) donde aparece claramente marcada la estacionalidad de rendimiento apícola, se puede observar que tiene dos máximos, uno se corresponde aproximadamente con el primer máximo de Ap, pero extendiéndose dos o tres meses más y apareciendo el mínimo de verano algo atrasado con respecto al correspondiente de Ap en los meses de agosto y septiembre y el segundo máximo en los meses de noviembre-diciembre, coincidiendo con el segundo mínimo (invernal) de Ap. Interpretamos esto como un desfasamiento entre los fenómenos, esto es, el resultado reflejado en la productividad melífera (momento de recolección) se retrasa dos o tres meses con respecto al proceso geofísico.

Consideramos que el conocimiento que nos proporciona el resultado analizado anteriormente puede constituir una útil herramienta para la mejor planificación a mediano plazo de la explotación apícola, independientemente de los ritmos seculares multianuales presentes en el fondo del proceso productivo, todo lo cual, aparejado a un mejor conocimiento de la variabilidad climática presente y los pronósticos meteorológicos en el plazo de meses puede aún contribuir más a obtener producciones mayores a más bajo costo.

La Tabla 1 nos permite discernir cuales son los períodos que con mayor frecuencia aparecen en cada mes y su coincidencia entre el índice Ap y la producción melífera. Es posible observar períodos que aparecen en ambas tablas para un número de meses considerable, lo cual se visualiza también en la Fig. 4, en la que se observa meses en que la variabilidad de la serie te tiempo es baja, sin embargo otros en que es alta como promedio para toda la muestra, o sea que para cada época del año el rendimiento apícola, como promedio, tiene un comportamiento típico que no se debe olvidar al trazar las estrategias de planificación del esfuerzo productivo.

Por último, la Fig. 8 nos ofrece un resultado global para los valores promedios anuales mostrando la regresión lineal ajustada a la asociación entre las variables Ap y Rendimiento. No resulta un coeficiente de correlación muy alto $(+0,53$ y SD $=0,13)$, pero que sí permite afirmar que existe una asociación multianual entre ambas variables. Para la producción de Miel y de Cera también se obtuvo esta regresión pero con valores de correlación algo más bajos. Un análisis de correlación cruzada pudiera mostrar más altos índices si tenemos en cuenta el desfasamiento entre las variables analizadas.

El resultado resumido en las Fig. 9 y 10 nos está expresando, entre otros detalles aún no analizados suficientemente, que dos a tres meses posteriores a los períodos (promedios mensuales y meses con Ap excepcionalmente altos) altos de actividad geomagnética, la producción y rendimiento se ven incrementados en más de una sigma sobre los valores promedios de los 10 meses vecinos, lo que nos puede proporcionar también un criterio a tener en cuenta en la planificación productiva a corto plazo. 
Recomendamos que, para un mejor conocimiento del fenómeno abordado en el presente trabajo, el cual confirma resultados precedentes, se lleve a cabo, donde exista una base de datos suficientemente extensa, análisis semejantes que puedan permitir una generalización y su posterior aplicación dentro de las estrategias de enfrentamiento a la variabilidad y cambio climático, lo cual, sin dudas redundaría en una mayor eficiencia productiva.

\section{Referencias Bibliográficas}

Babayev E.S. Crosby N.B., Obridko V.N. \& Rycroft M.J. (2008): Potential effects of solar and geomagnetic variability on terrestrial biological systems. Shamakhy Astrophysical Observatory (ShAO) named after N.Tusi\& Laboratory of Heliobiology, Baku, Azerbaijan National Academy of Sciences, 10.

E.W. Cliver, Y. Kamide, A.G.(2000) Ling. Mountains versus valleys'. Semiannual variation of geomagnetic activity.JOURNAL OF GEOPHYSICAL RESEARCH, VOL. 105, NO. A2, PAGES 2413-2424, FEBRUARY $1,2000$.

E.W. Cliver, Y. Kamide, A.G. Ling. (2002) The semiannual variation of geomagnetic activity: phases and profiles for 130 years of "aa" data. Journal of Atmospheric and Solar-Terrestrial Physics 64 (2002) 47-53

Pérez A., Sierra P., (1993). Preliminary Resultats About Possible Relation Between Heliomagnetical Disturbances and Honey Producction in Cuba. APIMONDIA. $33^{\text {rd }}$ International Apicultural Congress, Beijing, China, Set. $/ 1993$.

Sierra F.P., Sierra F.S., Rodríguez T. R., Pérez P. A. (1999): Impacto Medioambiental de las Perturbaciones Heliogeofísicas. Consideraciones a partir de Resultados Observacionales. Rev. Geofísica, No. 50, Enero-Junio 1999, pp. 9-23. México, D.F.

Sierra P., Pérez A., Durán O., Castellanos B., Zaldívar J.M. (2015). Aspectos a valorar dentro de la bioeconomía y su sendero de eco - intensificación para el buen desempeño de la actividad apícola ante la variabilidad de la Actividad Solar y Geomagnética y los cambios climáticos. VOL. 1, NÚM. 1 (2015). ISSN 2410- 7980, Copyright @ Rev. iberoam. bioecon. cambio clim. (Colegio Postgraduados y UNAN-León).

Sierra P., Baca M. (2013). Estado del Arte de la Bioeconomía y el Cambio Climático, Cap.3.3 Solar Activity, Agricultural Activity and Climate. Possible links. IBERO- AMERICAN PROGRAMME FOR SCIENCE, TECHNOLOGY AND DEVELOPMENT IBERO-AMERICAN NETWORK OF BIOECONOMICS AND 